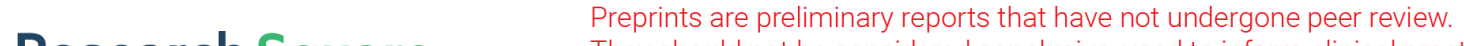 They should not be considered conclusive, used to inform clinical practice, or referenced by the media as validated information. \\ The Effect of Lysophosphatidic Acid-supplemented Culture Medium on Human Immature Oocytes Matured in Vitro
}

\section{Qigui Xie}

Shanghai Tenth People's Hospital, Nanjing Medical University

\section{Yaxin Xing}

Shanghai Tenth People's Hospital, Nanjing Medical University

Jianhong Zhou

Shanghai Tenth People's Hospital, Nanjing Medical University

\section{Ling Wang}

Shanghai Tenth People's Hospital, Nanjing Medical University

\section{Jie Wu}

The First Affiliated Hospital With Nanjing Medical University/Jiangsu Province Hospital, Nanjing

Medical University

\section{Richeng Chian ( $\nabla$ rchian@126.com )}

Shanghai 10th People's Hospital of Tongji University https://orcid.org/0000-0002-9938-1354

\section{Research}

Keywords: Oocytes, cumulus cells, in vitro maturation, lysophosphatidic acid, fertility

Posted Date: February 12th, 2021

DOl: https://doi.org/10.21203/rs.3.rs-196908/v1

License: (c) (1) This work is licensed under a Creative Commons Attribution 4.0 International License. Read Full License 


\section{Abstract}

Background: Lysophosphatidic acid-supplemented culture medium significantly increases the oocyte maturation rate in vitro. However, potential targets and pathways involved remain unknown.

Methods: A total of 43 women, who underwent cesarean section and aged between 18-35 years with good health, were included in this study. Immature oocytes were obtained and cultured with $10 \mathrm{mM}$ lysophosphatidic acid. After culture, oocyte maturation was assessed and oocytes and cumulus cells were collected for RNA sequencing. HISAT2 was used to align clean reads to the human genome. The featureCounts and edgeR package were used to calculate gene expression and analyze differences between groups respectively. ClusterProfiler program was used to perform Gene Ontology and Kyoto Encyclopedia of Genes and Genomes analysis.

Results: Oocyte maturation rate increased significantly after $48 \mathrm{~h}$ treatment with lysophosphatidic acid. In cumulus cells, 259 genes were upregulated and 237 were downregulated in the treatment group; Gene Ontology analysis revealed that 653 biological process, 61 cellular component, and 86 molecular function items were enriched by upregulated genes; 379 biological process, 46 cellular component, and 97 molecular function items were enriched by downregulated genes; Kyoto Encyclopedia of Genes and Genomes analysis showed that upregulated genes in the treatment group were enriched in TNF signaling and insulin secretion pathways and downregulated genes were enriched in TNF signaling and cell adhesion molecules. In oocytes, 128 genes were upregulated and downregulated in the treatment group; Gene Ontology analysis revealed that 300 biological process items, 44 cellular component items, and 93 molecular function items were enriched by upregulated genes; 268 biological process items, 22 cellular component items, and 72 molecular function items were enriched by downregulated genes; Kyoto Encyclopedia of Genes and Genomes analysis showed that upregulated genes in the treatment group were enriched in MAPK signaling, gap junction, and cell cycle pathways and downregulated genes were enriched in MAPK signaling, estrogen signaling, RAP1 signaling, and gap junction pathways.

Conclusions: Our study indicates that lysophosphatidic acid in culture medium enhances human oocyte maturation in vitro and identifies some potential targets and pathways associated with oocyte maturation for the first time and are important in clinical settings.

\section{Introduction}

The source of oocytes is the key issue for assisted reproductive technologies (1). There are different ways to obtain in vivo matured oocytes for infertility treatment. Normally, women are given ovarian stimulation to retrieve mature oocytes (2). However, ovarian stimulations with exogenous gonadotropin are accompanied by direct and indirect side-effects, including life-threatening ovarian hyper-stimulation syndrome (Feinberg, 2020). Therefore, mild ovarian stimulation protocols are promoted not only for safety but also their efficiency in developing assisted reproductive technologies (3). 
One option to avoid ovarian hyper-stimulation syndrome is to retrieve immature oocytes followed by in vitro maturation (IVM) (4). Several thousands of healthy babies have been born following IVM for women who were infertile with polycystic ovary syndrome (5), suggesting that mature and immature oocyte retrieval followed by in vitro fertilization/IVM could be an efficient infertility treatment for women who are under 35 years old with normal ovaries (6). IVM may also play an important role in fertility preservation for women before cancer treatment, because cell toxic cancer treatment may cause the loss ovarian function $(7,8)$.

Earlier studies indicated that immature oocytes can be retrieved during cesarean section and the obtained immature oocytes can be matured in vitro (9). Those immature oocytes may be used for in vitro studies and potentially preserved for fertility preservation (10-12). Although studies have reported that the quality of in vitro matured oocytes are affected by different culture media and supplements, it is still unclear how some factors promote oocyte maturation in the culture medium (13-17).

Lysophosphatidic acid (LPA) is a membrane phospholipid metabolite with growth factor- and hormonelike effects, and it is present in the human follicular fluid at a concentration of $10-25 \mu \mathrm{M}$ (18). Numerous studies have shown that LPA promotes oocyte maturation in vitro, as well as fertilization and embryonic development in animals, such as cattle, pig and mouse (19-21). However, there are few studies on the effect of LPA in the culture medium on human immature oocytes matured in vitro.

The cumulus-oocyte complex (COC) regulates the growth and development of mammalian oocytes and adjacent cumulus cells, and is believed to be the site of LPA synthesis and action (22). In this study, we investigated the effect of LPA in the culture medium on human immature oocyte, and the expression profiles of cumulus cells and oocytes. We aimed to reveal important signaling pathways and molecular mechanisms by which LPA stimulates oocyte maturation in vitro.

\section{Materials And Methods}

\section{Patients}

The inclusion criteria were as follows: Women who underwent cesarean section in Shanghai Tenth People's Hospital between November 1st, 2016 and March 31st, 2018; were aged between 18 and 35 years with good health; had not received any medication during pregnancy. Patients were randomly assigned to the control group and LPA treatment group. The enrolled patients gave their informed consent to be included in the study, which received the approval of the Ethics Review Committee of Shanghai Tenth People's Hospital (No. SHSY-IEC-1.0/16 - 03/01).

\section{IVM culture media}

The control group culture medium comprised of $75 \mathrm{mIU} / \mathrm{mL}$ follicle-stimulating hormone, $75 \mathrm{mlU} / \mathrm{mL}$ luteinizing hormone, and $10 \mathrm{ng} / \mathrm{mL}$ human epidermal growth factor. The LPA group culture medium 
comprised of $75 \mathrm{mlU} / \mathrm{mL}$ follicle-stimulating hormone, $75 \mathrm{mlU} / \mathrm{mL}$ luteinizing hormone, $10 \mathrm{ng} / \mathrm{mL}$ human epidermal growth factor, and $10 \mu \mathrm{M}$ LPA.

\section{Acquisition and culture of immature oocytes}

Once the uterus had been sutured during the cesarean section, antral follicles were aspirated using a 5$\mathrm{mL}$ syringe. The syringes were transferred to the laboratory, and follicular fluid was poured to Petri dishes to look for COCs. COCs with at least three layers of tightly packed cumulus cells were transferred to culture medium for maturation. COCs from each patient were randomly allocated to one of the two groups: control and LPA treatment groups. COCs were cultured in a two-well dish; the inner well contained $1 \mathrm{~mL}$ of IVM medium, and the outer-well contained $2 \mathrm{~mL} \mathrm{IVM}$ medium. COCs were cultured in an incubator containing $5 \% \mathrm{CO}_{2}$ and $5 \% \mathrm{O}_{2}$ at $37^{\circ} \mathrm{C}$. Following $24 \mathrm{~h}$ and $48 \mathrm{~h}$ of culture, COCs were placed into hyaluronidase solution ( 80 units $/ \mathrm{mL}$ ) for 1 min and the surrounding cumulus cells were mechanically denuded to assess the oocytes maturity. The mature oocyte (metaphase-II) was identified via the extrusion of the first polar body. Six mature COCs from three patients were donated for molecular analysis and the denuded cumulus cells and mature oocytes were collected separately.

\section{RNA extraction, library preparation, and sequencing}

A Smart-Seq2 library was prepared as previously described (23). Briefly, we performed single-cell sorting, cell lysis, reverse transcription, and cDNA synthesis. The library was constructed using an Illumina Nextera kit and size-selected using AMPure XP beads and examined via quantitative PCR for sequencing. A NovaSeq 6000 System (Illumina, San Diego, CA, USA) was used for sequencing with a PE150 read length. The data were deposited at the National Center for Biotechnology Information with an assigned No. PRJNA678410.

\section{Expression profile analysis}

Clean reads were obtained using the Cutadapt software to remove reads containing adaptors and lowquality reads (over $50 \%$ of reads with Q values $\leq 30$ ). HISAT2 was used to align the clean reads to the human genome. The featureCounts program was used to calculate gene expression, and the edgeR package was used to analyze differences between groups. Genes with $p<0.05$ and |log2FoldChange| values $>1$ were defined as differentially expressed genes.

\section{Gene ontology (GO) and Kyoto Encyclopedia of Genes and Genomes (KEGG) analysis}

The clusterProfiler program was used to perform GO analysis, KEGG analysis and select false discovery rates of $<0.05$ to determine pathways enriched by differentially expressed genes $(p<0.05)$.

\section{Results}

Effect of LPA treatment on human immature oocyte maturation in vitro 
A total of 155 immature oocytes were obtained. After $48 \mathrm{~h}$ of culture, there were 65 out of 74 mature oocytes in the LPA treatment group and 61 out of 81 mature oocytes in the control group. The maturation rate was significantly higher $(P<0.05)$ in the LPA treatment group $(87.8 \%)$ compared to that in the control group (75.3\%) (Fig. 1).

\section{LPA treatment altered the expression profiles in cumulus cells and oocytes}

There were significant differences in expression profiles of control and LPA treatment groups. In cumulus cells, 259 genes were upregulated and 237 genes were downregulated in the LPA treatment group compared to the control group (a total of 496 genes). In oocytes, 128 genes were upregulated and 128 genes were downregulated in the LPA treatment group compared to the control group (a total of 256 genes). Among them, 248 differentially expressed genes were unique to the LPA treatment group (Fig. 2A). Heatmaps clearly showed that LPA treatment and control groups could be classified into different clusters (Fig. 2B and 2C).

\section{LPA treatment led to differences in the enrichment of functional items between cumulus cells and oocytes.}

In cumulus cells, GO enrichment analysis of upregulated and downregulated genes revealed that 653 biological process (BP), 61 cellular component (CC), and 86 molecular function (MF) items were enriched by upregulated genes, and 379 BP, 46 CC, and 97 MF items were enriched by downregulated genes. The top 20 GO-derived items enriched by differentially expressed genes were obtained. The upregulated genes that affected BPs were involved in the import, secretion, localization, and transport of proteins and in the biosynthesis and regulation of chemokines, and those that affected CCs were associated with extracellular substances and components related to the cell periphery and plasma membrane (Fig. 3A). In contrast, the top 20 GO-derived items enriched by downregulated genes that affected BPs were involved in the mitotic cell cycle and androgen metabolism, and those that affected CCs were involved in the cell periphery and transmembrane transporter complexes (Fig. 3B).

In oocytes, GO analysis revealed that 300 BP items, 44 CC items, and 93 MF items were enriched by upregulated genes, and 268 BP items, 22 CC items, and 72 MF items were enriched by downregulated genes. The top $20 \mathrm{GO}$-derived items enriched by genes that were differentially expressed were also revealed. The upregulated genes that affected BPs were involved in biosynthetic processes and cell-cell adhesion, and those that affected CCs were involved with the sperm connecting piece and the cell-cell junction (Fig. 3C). In contrast, the downregulated genes that affected BPs were involved in cell differentiation, dGMP metabolism, and dGDP biosynthesis, and those that affected CCs were associated with cell periphery (Fig. 3D).

KEGG pathway analysis enabled the comparison of pathways enriched by differentially expressed genes. In cumulus cells, the upregulated genes in the LPA treatment group were enriched in TNF signaling and insulin secretion pathways (Fig. 4A), and pathways enriched by downregulated genes included TNF 
signaling pathway and cell adhesion molecules (CAMs) pathway (Fig. 4A). The TNF signaling pathway was enriched by both the upregulation and downregulation of certain differentially expressed genes.

In oocytes, the upregulated genes in the LPA treatment group were enriched in MAPK signaling, gap junction, and cell cycle pathways (Fig. 4B). The downregulated genes in the LPA treatment were enriched in MAPK signaling, estrogen signaling, RAP1 signaling, and gap junction pathways. MAPK signaling and gap junction pathways were enriched by both upregulated and downregulated genes (Fig. 4B).

\section{Discussion}

We studied the effects of LPA on human immature oocytes and changes in the gene expression profiles in cumulus cells and oocytes during in vitro culture. There were significant differences in the gene expression profile between LPA treatment and control groups. The differences might explain the functional role of LPA in the culture medium to promote oocyte maturation, as well as the molecular mechanisms behind the phenotype.

LPA is present in the human follicular fluid at a concentration of 10-25 $\mu \mathrm{M}$ (Tokumura et al., 1999). In our study, we chose $10 \mu \mathrm{M}$ of LPA as the final concentration in the culture medium based on previous studied on a mouse model and our previous study, in which we reported that oocyte maturation rates were significantly higher in $10 \mu \mathrm{M}$ LPA-treated group than the control (Komatsu et al., 2006). Studies have shown that SMAD4 plays an important role during the development of oocytes from small antral follicles (1-3 $\mathrm{mm}$ in diameter) to large antral follicles (3-7 mm in diameter) (Komatsu et al., 2006). In a study by $\mathrm{Li}$ et al., the litter size was reduced in mice lacking Smad4 compared to the control mice (24). In the present study, the expression of this gene was upregulated in oocytes treated with LPA, indicating it may involve into the maturation of oocytes in vitro.

The biosynthetic function of ribosomes is a critical factor for the development of embryos (25). In this study, upregulated genes enriched the GO-derived items $\mathrm{BP}, \mathrm{CC}$, and MF, which are related to ribosome entry in the LPA-treated cumulus cells. In the control group, the top 20 GO-derived items enriched by upregulated genes were involved in the import, secretion, localization, and transport of eight proteins and in the biosynthesis and regulation of two chemokines. This implies that LPA treatment may stimulate the oocyte maturation in vitro.

Interleukin 6 (IL6) can mediate tyrosine kinase receptor A to regulate cumulus expansion, and IL6 knockout likewise reduces cumulus expansion (26). Insulin-like growth factor 1 (IGF1) enables cumulus cells to synthesize and accumulate hyaluronic acid, thereby promoting cumulus cell expansion (27). IL6 and IGF1 enriched eight items related to the import, secretion, localization, and transport of proteins, as well as the biosynthesis and regulation of chemokines, indicating that IL6 and IGF1 were key to the promotion of human oocyte maturation by LPA. The upregulated gene encoding heme oxygenase 1 (HMOX1) enriched two biosynthetic items related to chemokines. Studies have shown that the expression of this gene in cumulus cells can be used as a biomarker of oocyte compatibility (28). Downregulated 
BPs include processes relating to androgen metabolites, and androgens can promote the expression of connexin 37 (CX37) in COCs, thereby eliciting an ovarian response (29).

Studies by Gebhardt et al. have shown that high levels of PTGS2 expression are related to the rate of live birth (30). Moreover, high levels of PTGS2 expression in the cumulus cells surrounding mature oocytes are related to high-quality embryos and embryo sacs (31). In the present study, PTGS2 expression decreased in the cumulus cells of LPA treatment group compared to that in the control group, which suggested that PTGS2 negatively regulated the development of LPA-treated oocytes. However, the specific function of PTGS2 and the mechanism by which it acts require further investigation. In this study, transcriptome analysis revealed that only four differentially expressed genes were upregulated in the cumulus cells and only four were downregulated in oocytes, indicating that LPA had varying effects on the gene expression of cumulus cells and oocytes.

KEGG pathway enrichment analysis revealed upregulated and downregulated genes in the cumulus cells that enrich the TNF signaling pathway, and the cumulus cells can release soluble TNF-a to promote oocyte aging (32). In our study, the upregulated genes in the cumulus cells enriched the insulin secretion pathway. Insulin plays a central role in polycystic ovary syndrome, and it engages with the IGF1 receptor to enhance steroid production in ovaries and adrenal glands (33). Downregulated differentially expressed genes enrich signaling pathways related to CAMs and AMPK. In the cumulus cells, the downregulation of CAMs may cause polycystic ovary syndrome (34).

Among the top 20 GO-derived items enriched by upregulated genes in the oocytes of the LPA-treated group, five were related to biosynthetic processes and one was related to cell-cell adhesion. Both PLEK and PRKG1 enriched these six items. The expression of PLEK may promote cell growth and development (35), and PRKG1 may be related to early life adversity (36). The present results suggested that PLEK and $P R K G 1$ were important for the maturation and development of oocytes in the culture medium supplemented with LPA, but there have not been any reports on these two genes regarding human oocyte maturation. We found that 47 downregulated genes enriched CC items related to the cell periphery; it is interesting to note that chromosomes separate during cell division at the cell periphery (37). Thus, our results indicated that LPA might affect cell division.

In the oocytes, both upregulated and downregulated genes enriched items related to the MAPK signaling pathway and the gap junction. MAPK signaling pathway regulates the development of oocytes (38). During the development of COCs, the gap junction pathway mediates material exchange between oocytes and cumulus cells (39). Downregulated genes enriched the estrogen signaling pathway and the RAP1 signaling pathway. Estrogen affects the physiological development of women and plays an important role in the maturation of oocytes (40). The RAP1 signaling pathway in oocytes involves multiple cellular processes, including secretion, cell adhesion, and intercellular junction formation, and regulates oocyte maturation and embryonic development (41).

There are limits in our study. Due to the limited number of participants and available immature oocytes, we had a relatively small number of cumulus cells and oocytes for RNA sequencing. If possible in clinics, 
we will increase the sample size in future studies.

\section{Conclusions}

We found that oocyte maturation is promoted by LPA in the culture medium and identified some potential targets and pathways associated with oocyte maturation in vitro for the first time. Our findings have important clinical significance and warrant further functional studies.

\section{Abbreviations}

IVM: in vitro maturation

LPA: lysophosphatidic acid

COC: cumulus-oocyte complex

GO: Gene ontology

KEGG: Kyoto Encyclopedia of Genes and Genomes

BP: Biological process

CC: Cellular component

MF: molecular function

CAMs: cell adhesion molecules

IL6: Interleukin 6

\section{Declarations}

\section{Ethics approval and consent to participate}

The enrolled patients gave their informed consent to be included in the study, which received the approval of the Ethics Review Committee of Shanghai Tenth People's Hospital (No. SHSY-IEC-1.0/16-03/01)

\section{Consent for publication}

Written informed consents for publication were obtained from the participants.

\section{Availability of data and materials}

The sequencing data were deposited at the National Center for Biotechnology Information with an assigned No. PRJNA678410 (https://www.ncbi.nlm.nih.gov/bioproject/PRJNA678410). 


\section{Competing interests}

The authors declare that they have no conflicts of interests.

\section{Funding}

This work was supported by the Ministry of Science and Technology of China (No. 2017YFC1001601) and National Key R\&D Program of China (No. 2017YFC1002003). The funding body was not involved in the study design, collection, analysis, data interpretation and manuscript writing.

\section{Author's contributions}

QX and YX carried out the experiments. JZ and LW analyzed the data. QX wrote the draft of manuscript. JW and RC designed the study and revised the manuscript. All authors have read and agreed to the publicized version of the manuscript.

\section{Acknowledgements}

$\mathrm{N} / \mathrm{A}$

\section{References}

1. Christianson MS, Bellver J. Innovations in assisted reproductive technologies: impact on contemporary donor egg practice and future advances. Fertility and sterility. 2018;110(6):994-1002.

2. Pacchiarotti A, Selman H, Valeri C, Napoletano S, Sbracia M, Antonini G, et al. Ovarian Stimulation Protocol in IVF: An Up-to-Date Review of the Literature. Curr Pharm Biotechnol. 2016;17(4):303-15.

3. Nargund G, Fauser B. Mild ovarian stimulation for IVF is the smartest way forward. Reproductive biomedicine online. 2020;41(4):569-71.

4. Walls ML, Hart RJ. In vitro maturation. Best practice \& research Clinical obstetrics \& gynaecology. 2018;53:60-72.

5. Yang ZY, Chian RC. Development of in vitro maturation techniques for clinical applications. Fertility and sterility. 2017;108(4):577-84.

6. Yang SH, Patrizio P, Yoon SH, Lim JH, Chian RC. Comparison of pregnancy outcomes in natural cycle IVF/M treatment with or without mature oocytes retrieved at time of egg collection. Systems biology in reproductive medicine. 2012;58(3):154-9.

7. Creux H, Monnier P, Son WY, Buckett W. Thirteen years' experience in fertility preservation for cancer patients after in vitro fertilization and in vitro maturation treatments. Journal of assisted reproduction and genetics. 2018;35(4):583-92.

8. Shirasawa $H$, Terada $Y$. In vitro maturation of human immature oocytes for fertility preservation and research material. Reproductive medicine and biology. 2017;16(3):258-67. 
9. Cha KY, Chian RC. Maturation in vitro of immature human oocytes for clinical use. Human reproduction update. 1998;4(2):103-20.

10. Ben-Haroush A, Sapir O, Fisch B. Aspiration of immature oocytes during cesarean section for fertility preservation and future surrogacy. American journal of obstetrics and gynecology. 2010;203(1):e124.

11. Pongsuthirak P, Vutyavanich T. Developmental competence of in vitro-matured human oocytes obtained from pregnant and non-pregnant women. Clinical and experimental reproductive medicine. 2018;45(4):189-94.

12. Pongsuthirak P, Songveeratham S, Vutyavanich T. Comparison of blastocyst and Sage media for in vitro maturation of human immature oocytes. Reproductive sciences. 2015;22(3):343-6.

13. Omidi M, Khalili MA, Ashourzadeh S, Rahimipour M. Zona pellucida birefringence and meiotic spindle visualisation of human oocytes are not influenced by IVM technology. Reproduction, fertility, and development. 2014;26(3):407-13.

14. Dal Canto M, Novara PV, Coticchio G, Mignini Renzini M, Brambillasca F, Brigante C, et al. Morphokinetics of embryos developed from oocytes matured in vitro. Journal of assisted reproduction and genetics. 2016;33(2):247-53.

15. Walls ML, Hart R, Keelan JA, Ryan JP. Structural and morphologic differences in human oocytes after in vitro maturation compared with standard in vitro fertilization. Fertility and sterility. 2016;106(6):1392-8 e5.

16. Chian RC, Buckett WM, Tan SL. In-vitro maturation of human oocytes. Reproductive biomedicine online. 2004;8(2):148-66.

17. Veitia RA. Primary ovarian insufficiency, meiosis and DNA repair. Biomedical journal. 2020;43(2):11523.

18. Tokumura A, Miyake M, Nishioka Y, Yamano S, Aono T, Fukuzawa K. Production of lysophosphatidic acids by lysophospholipase $D$ in human follicular fluids of In vitro fertilization patients. Biol Reprod. 1999;61(1):195-9.

19. Boruszewska D, Torres AC, Kowalczyk-Zieba I, Diniz P, Batista M, Lopes-da-Costa L, et al. The effect of lysophosphatidic acid during in vitro maturation of bovine oocytes: embryonic development and mRNA abundances of genes involved in apoptosis and oocyte competence. Mediators Inflamm. 2014;2014:670670.

20. Zhang JY, Jiang Y, Lin T, Kang JW, Lee JE, Jin DI. Lysophosphatidic acid improves porcine oocyte maturation and embryo development in vitro. Mol Reprod Dev. 2015;82(1):66-77.

21. Jo JW, Jee BC, Suh CS, Kim SH. Addition of lysophosphatidic acid to mouse oocyte maturation media can enhance fertilization and developmental competence. Hum Reprod. 2014;29(2):234-41.

22. Boruszewska D, Sinderewicz E, Kowalczyk-Zieba I, Skarzynski DJ, Woclawek-Potocka I. Influence of lysophosphatidic acid on estradiol production and follicle stimulating hormone action in bovine granulosa cells. Reprod Biol. 2013;13(4):344-7. 
23. Picelli S, Faridani OR, Bjorklund AK, Winberg G, Sagasser S, Sandberg R. Full-length RNA-seq from single cells using Smart-seq2. Nat Protoc. 2014;9(1):171-81.

24. Li X, Tripurani SK, James R, Pangas SA. Minimal fertility defects in mice deficient in oocyteexpressed Smad4. Biol Reprod. 2012;86(1):1-6.

25. Kim D, Paggi JM, Park C, Bennett C, Salzberg SL. Graph-based genome alignment and genotyping with HISAT2 and HISAT-genotype. Nat Biotechnol. 2019;37(8):907-15.

26. Wang $Y$, Liang N, Yao G, Tian H, Zhai Y, Yin Y, et al. Knockdown of TrkA in cumulus oocyte complexes (COCs) inhibits EGF-induced cumulus expansion by down-regulation of IL-6. Mol Cell Endocrinol. 2014;382(2):804-13.

27. Nagyova E. Regulation of cumulus expansion and hyaluronan synthesis in porcine oocyte-cumulus complexes during in vitro maturation. Endocr Regul. 2012;46(4):225-35.

28. Bergandi L, Basso G, Evangelista F, Canosa S, Dalmasso P, Aldieri E, et al. Inducible nitric oxide synthase and heme oxygenase 1 are expressed in human cumulus cells and may be used as biomarkers of oocyte competence. Reprod Sci. 2014;21(11):1370-7.

29. Zhang Y, Xu Y, Kuai Y, Wang S, Xue Q, Shang J. Effect of testosterone on the Connexin37 of sexual mature mouse cumulus oocyte complex. J Ovarian Res. 2016;9(1):82.

30. Gebhardt KM, Feil DK, Dunning KR, Lane M, Russell DL. Human cumulus cell gene expression as a biomarker of pregnancy outcome after single embryo transfer. Fertil Steril. 2011;96(1):47-52 e2.

31. Borgbo T, Povlsen BB, Andersen CY, Borup R, Humaidan P, Grondahl ML. Comparison of gene expression profiles in granulosa and cumulus cells after ovulation induction with either human chorionic gonadotropin or a gonadotropin-releasing hormone agonist trigger. Fertil Steril. 2013;100(4):994-1001.

32. Kong QQ, Wang J, Xiao B, Lin FH, Zhu J, Sun GY, et al. Cumulus cell-released tumor necrosis factor (TNF)-alpha promotes post-ovulatory aging of mouse oocytes. Aging (Albany NY). 2018;10(7):174557.

33. Chehin MB, Fraietta R, Lorenzon AR, Bonetti TCS, Motta ELA. The insulin signaling pathway is dysregulated in cumulus cells from obese, infertile women with polycystic ovarian syndrome with an absence of clinical insulin resistance. Ther Adv Reprod Health. 2020;14:2633494120906866.

34. Hassani F, Oryan S, Eftekhari-Yazdi P, Bazrgar M, Moini A, Nasiri N, et al. Downregulation of Extracellular Matrix and Cell Adhesion Molecules in Cumulus Cells of Infertile Polycystic Ovary Syndrome Women with and without Insulin Resistance. Cell J. 2019;21(1):35-42.

35. Guo J, Cai Y, Ye X, Ma N, Wang Y, Yu B, et al. MiR-409-5p as a Regulator of Neurite Growth Is Down Regulated in APP/PS1 Murine Model of Alzheimer's Disease. Front Neurosci. 2019;13:1264.

36. Struk AA, Mugon J, Huston A, Scholer AA, Stadler G, Higgins ET, et al. Self-regulation and the foraging gene (PRKG1) in humans. Proc Natl Acad Sci U S A. 2019;116(10):4434-9.

37. Carvalhal S, Stevense M, Koehler K, Naumann R, Huebner A, Jessberger R, et al. ALADIN is required for the production of fertile mouse oocytes. Mol Biol Cell. 2017;28(19):2470-8. 
38. Liu W, Zhan C, Zhang T, Zhang X. Microcystin-LR influences the in vitro oocyte maturation of zebrafish by activating the MAPK pathway. Aquat Toxicol. 2019;215:105261.

39. Acuna-Hernandez DG, Arreola-Mendoza L, Santacruz-Marquez R, Garcia-Zepeda SP, Parra-Forero LY, Olivares-Reyes JA, et al. Bisphenol A alters oocyte maturation by prematurely closing gap junctions in the cumulus cell-oocyte complex. Toxicol Appl Pharmacol. 2018;344:13-22.

40. Bilodeau-Goeseels S, Magyara N, Collignon C. Characterization of the effects of metformin on porcine oocyte meiosis and on AMP-activated protein kinase activation in oocytes and cumulus cells. Zygote. 2014;22(2):275-85.

41. Wang JC, Geng Y, Han Y, Luo HN, Zhang YS. Dynamic expression of Epac and Rap1 in mouse oocytes and preimplantation embryos. Exp Ther Med. 2018;16(2):523-8.

\section{Figures}

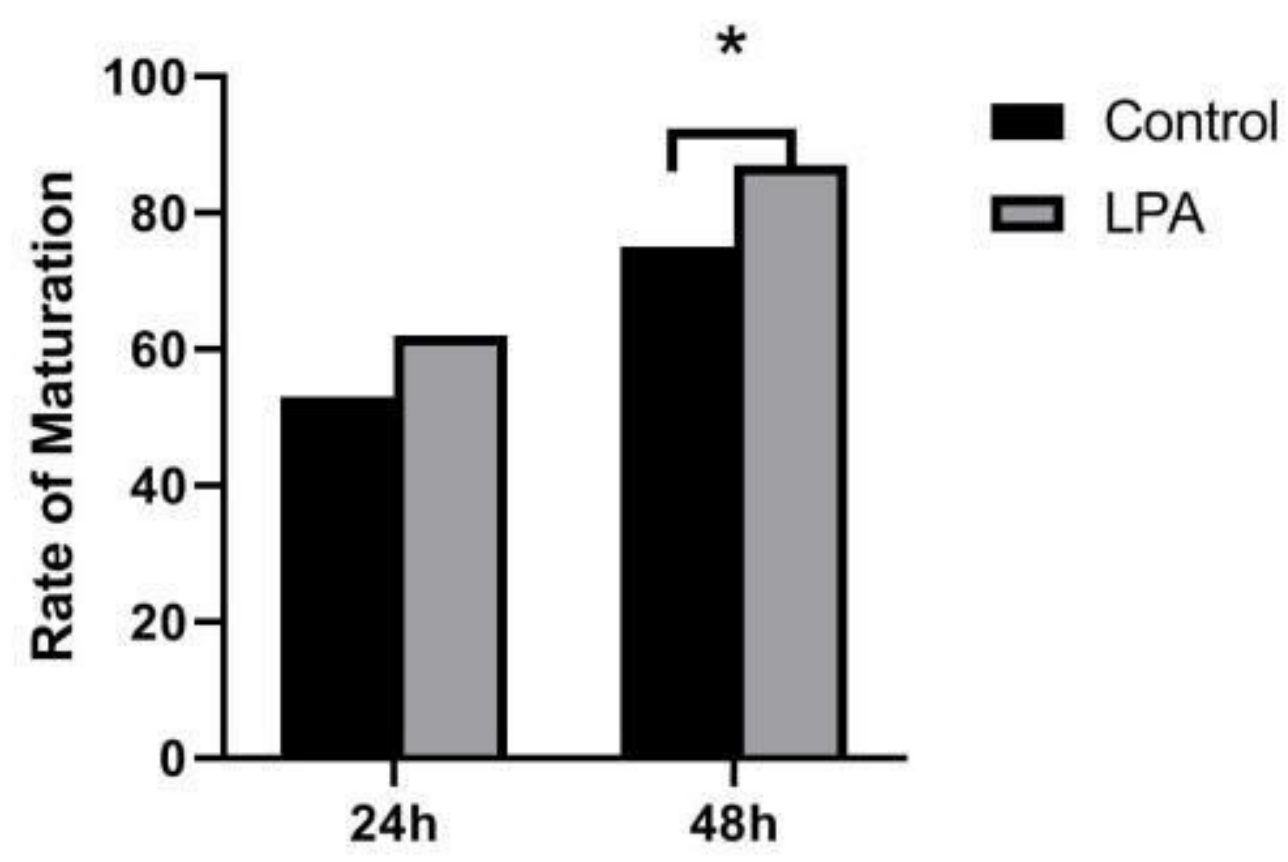

Figure 1

Maturity rate in the control group and LPA group after 24 and $48 \mathrm{~h}$. *Indicates significantly different between group $(P<0.05)$. LPA, Lysophosphatidic acid. 
A
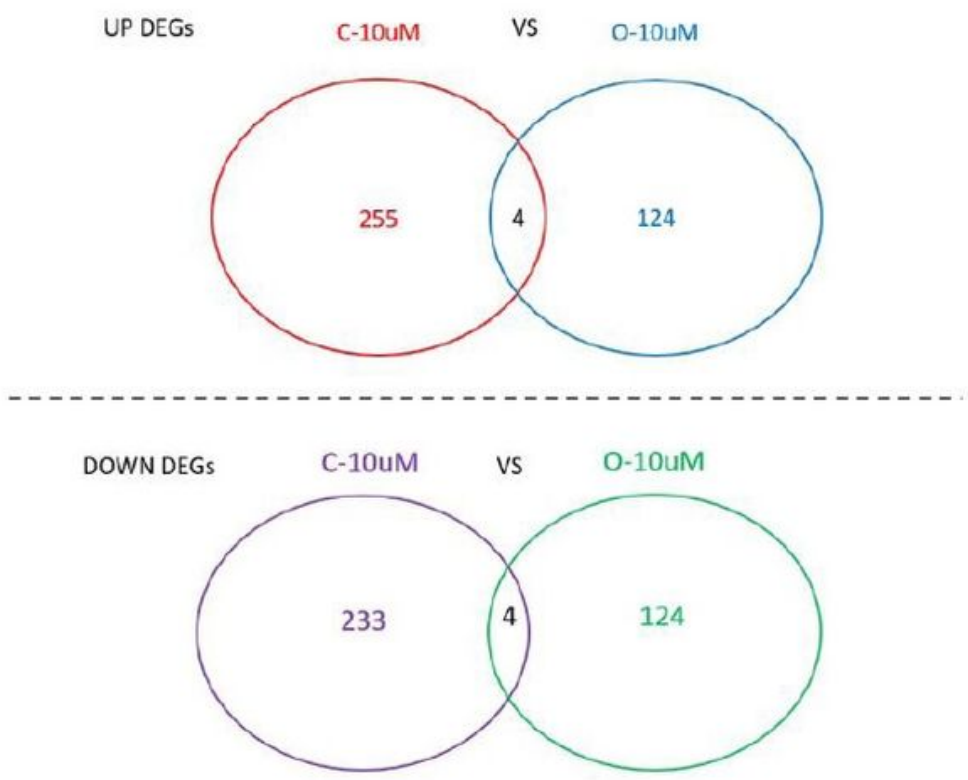

B

$\mathrm{C}$

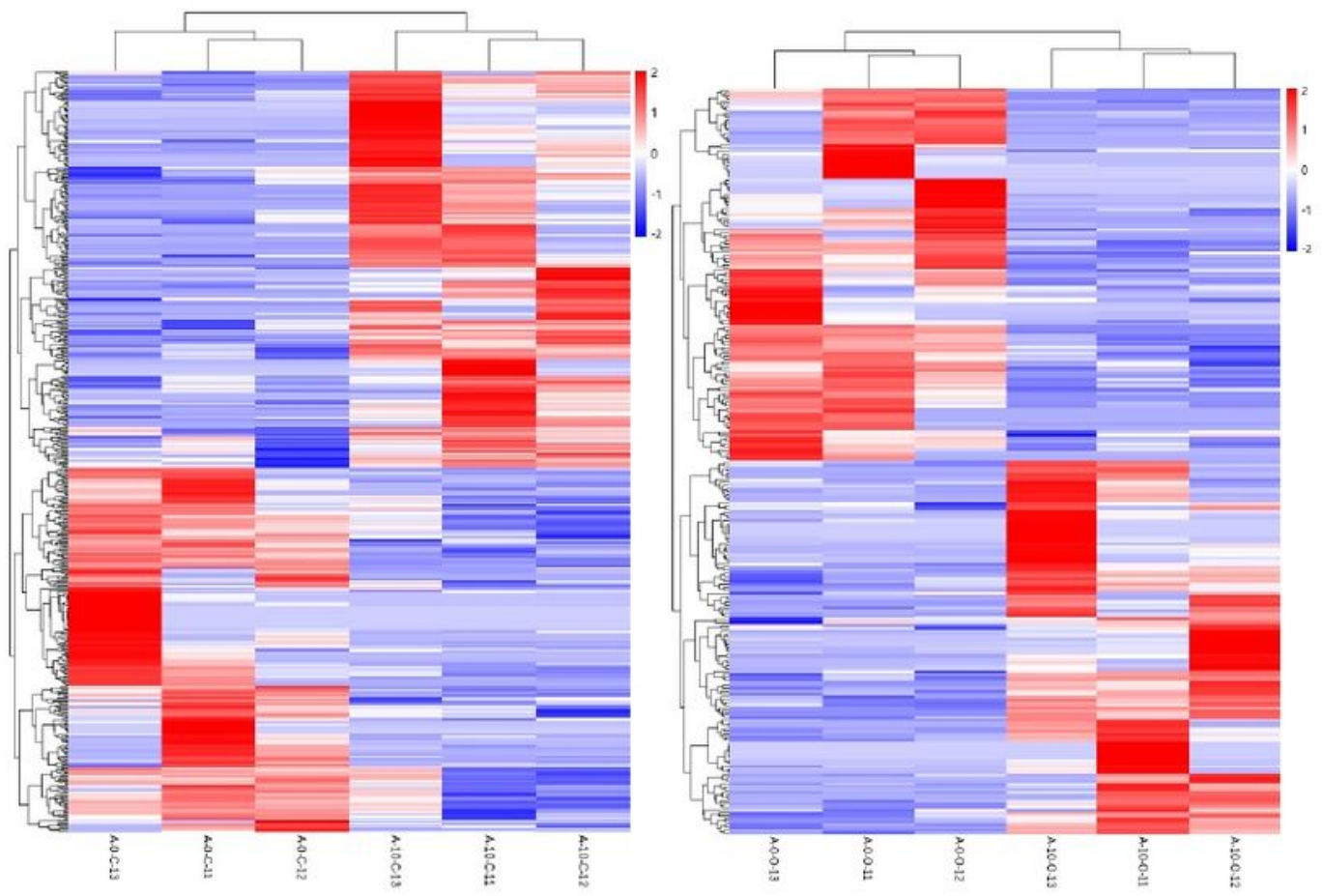

Figure 2

Venn diagram and heatmap of cumulus cells and oocytes between LPA-treated and control groups. $2 \mathrm{~A}$. VENN diagrams of differentially expressed genes between control and 0-10 $\mu \mathrm{M}$ groups and C-10 $\mu \mathrm{M}$ and control groups. Control group is the group without LPA treatment. 0-10 $\mu \mathrm{M}$ group is the group of oocytes treated with LPA at a concentration of $10 \mu \mathrm{M}$. C-10 $\mu \mathrm{M}$ group is the group of cumulus cells treated with LPA at a concentration of $10 \mu \mathrm{M}$. 2B. Heatmap of differentially expressed genes between C-Control group 
vs $\mathrm{C}-10 \mu \mathrm{M}$, in which $\mathrm{A}-\mathrm{-}-\mathrm{C}-11, \mathrm{~A}-\mathrm{O}-\mathrm{C}-12$ and $\mathrm{A}-0-\mathrm{C}-13$ were cumulus cells without LPA and A-10-C-11, A-10$\mathrm{C}-12$ and $\mathrm{A}-10-\mathrm{C}-13$ were cumulus cells treated with $10 \mu \mathrm{M}$ of LPA. 2C. Heatmap of differentially expressed genes between 0-Control group vs 0-10 $\mu \mathrm{M}$, in which A-0-0-11, A-0-0-12 and A-0-0-13 were oocytes without LPA and A-10-0-11, A-10-0-12 and A-10-0-13 were oocytes treated with $10 \mu \mathrm{M}$ of LPA. LPA, Lysophosphatidic acid.

A

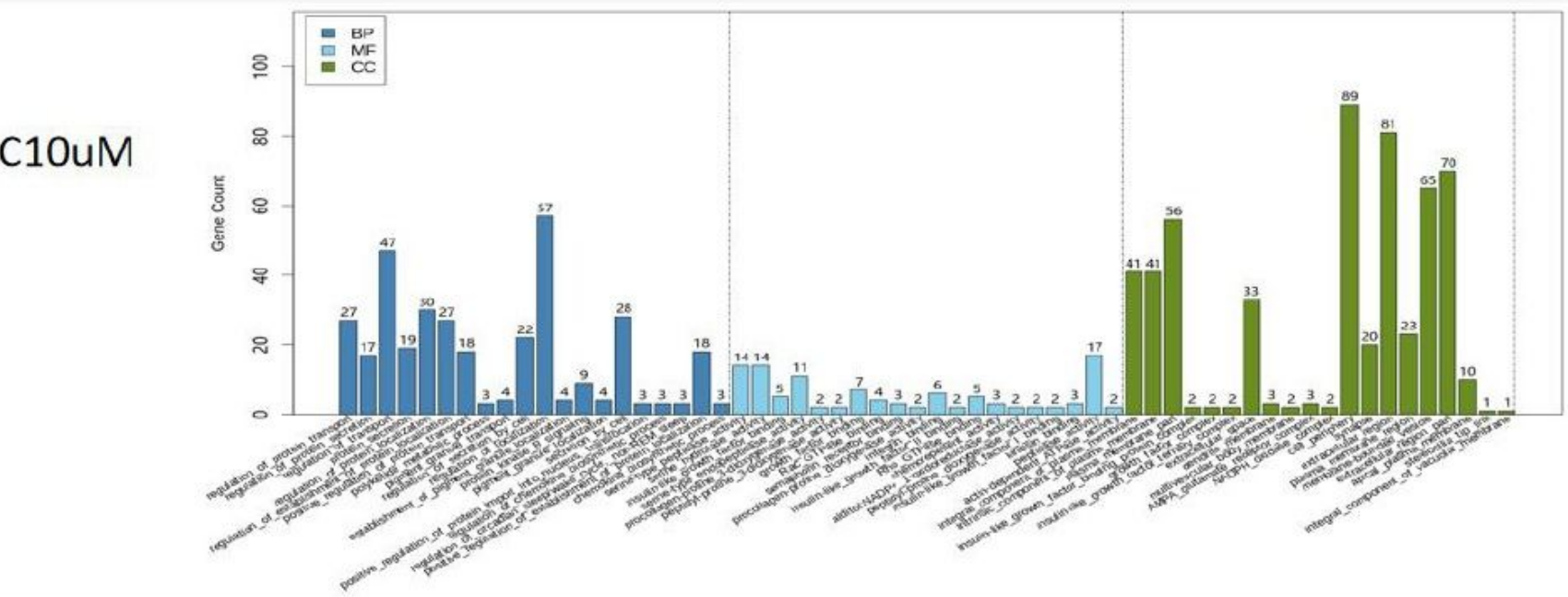

B

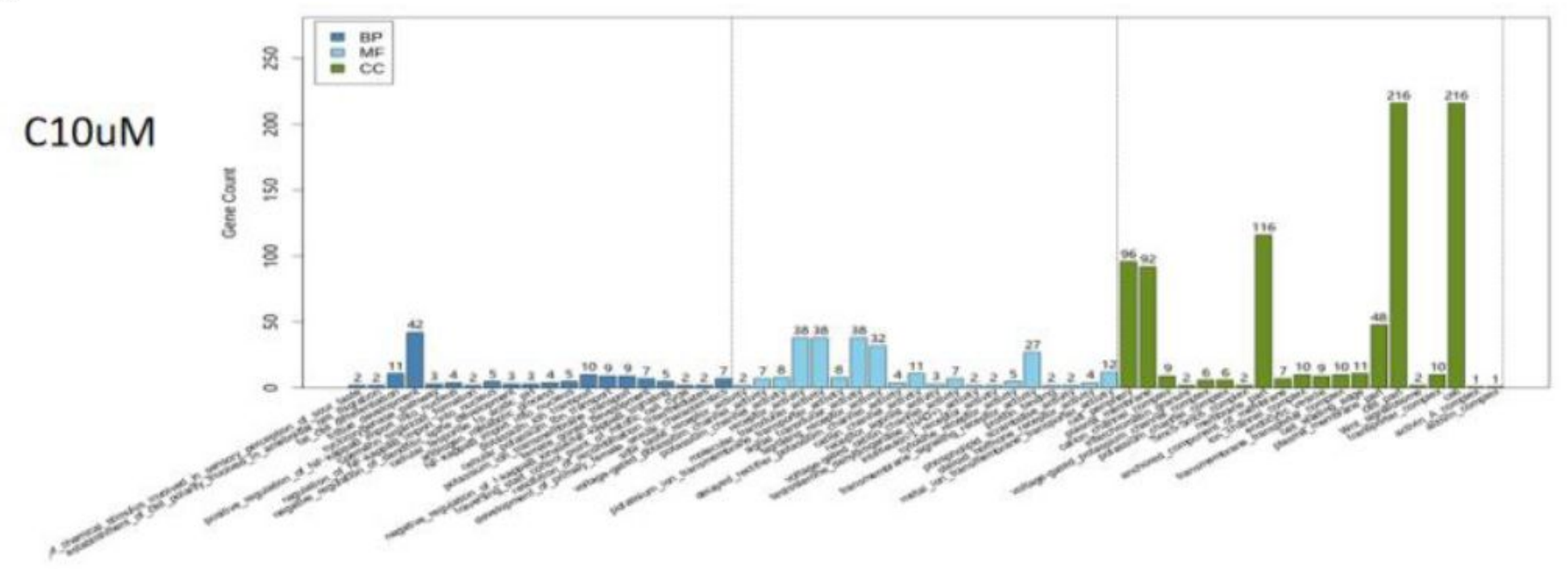

\section{Figure 3}

Top20 GO items enriched from differentially expressed genes in the 0-10 $\mu \mathrm{M}$ and C-10 $\mu \mathrm{M}$ group. 0-10 $\mu \mathrm{M}$ group is the oocytes group treated by $10 \mu \mathrm{M}$ of LPA and C-10 $\mu \mathrm{M}$ group is the cumulus cells treated with $10 \mu \mathrm{M}$ of LPA. 3A. Top20 GO items enriched from upregulated genes in the C-10 $\mu \mathrm{M}$ group; 3B. Top20 GO items enriched from downregulated genes in the $\mathrm{C}-10 \mu \mathrm{M}$ group; $3 \mathrm{C}$. Top20 $\mathrm{GO}$ items enriched from upregulated genes in the 0-10 $\mu \mathrm{M}$ group; 3D. Top20 GO items enriched from downregulated genes in the 0-10 $\mu \mathrm{M}$ group. LPA, Lysophosphatidic acid; GO, Gene Ontology. 
A.

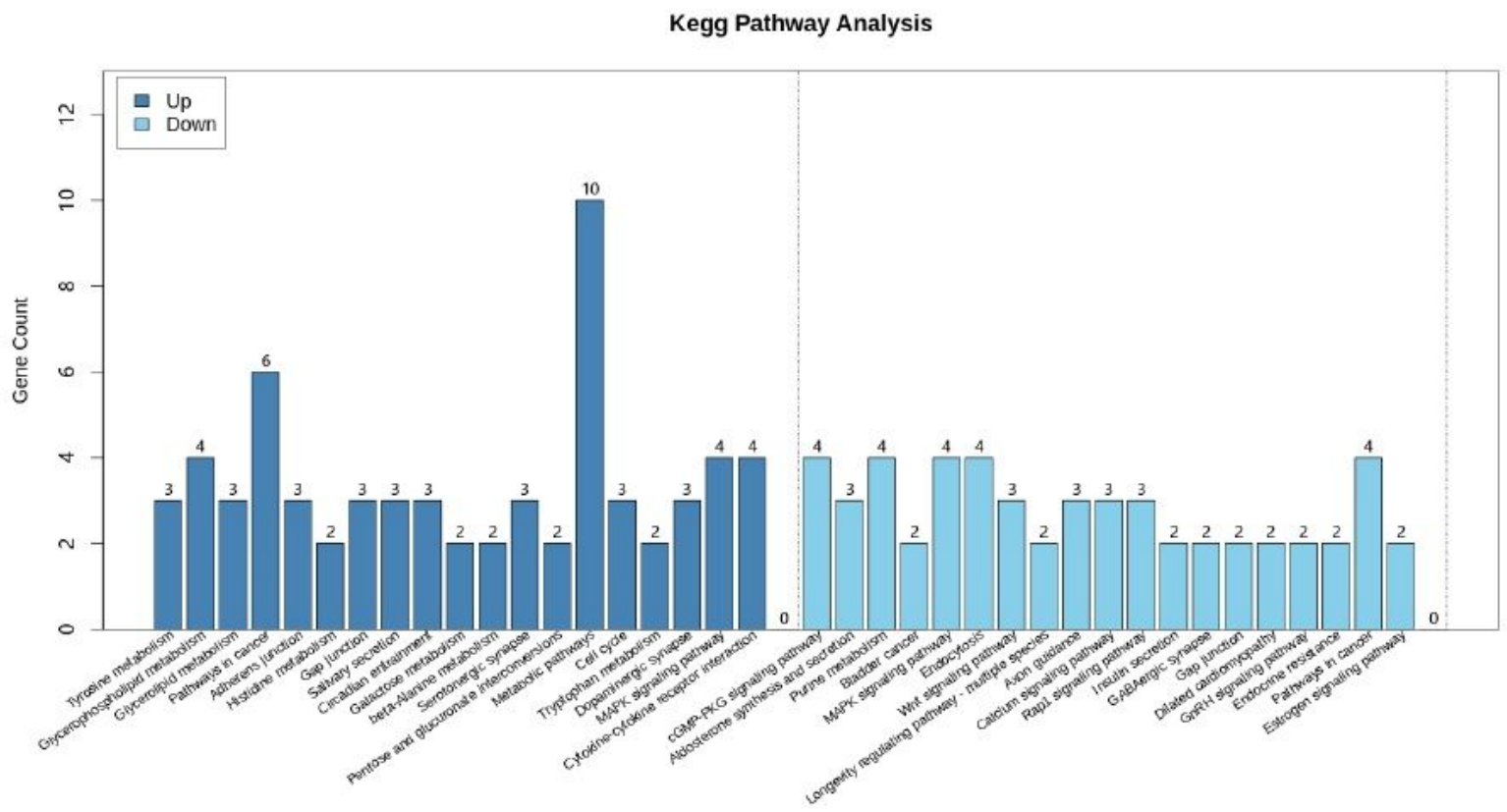

B.

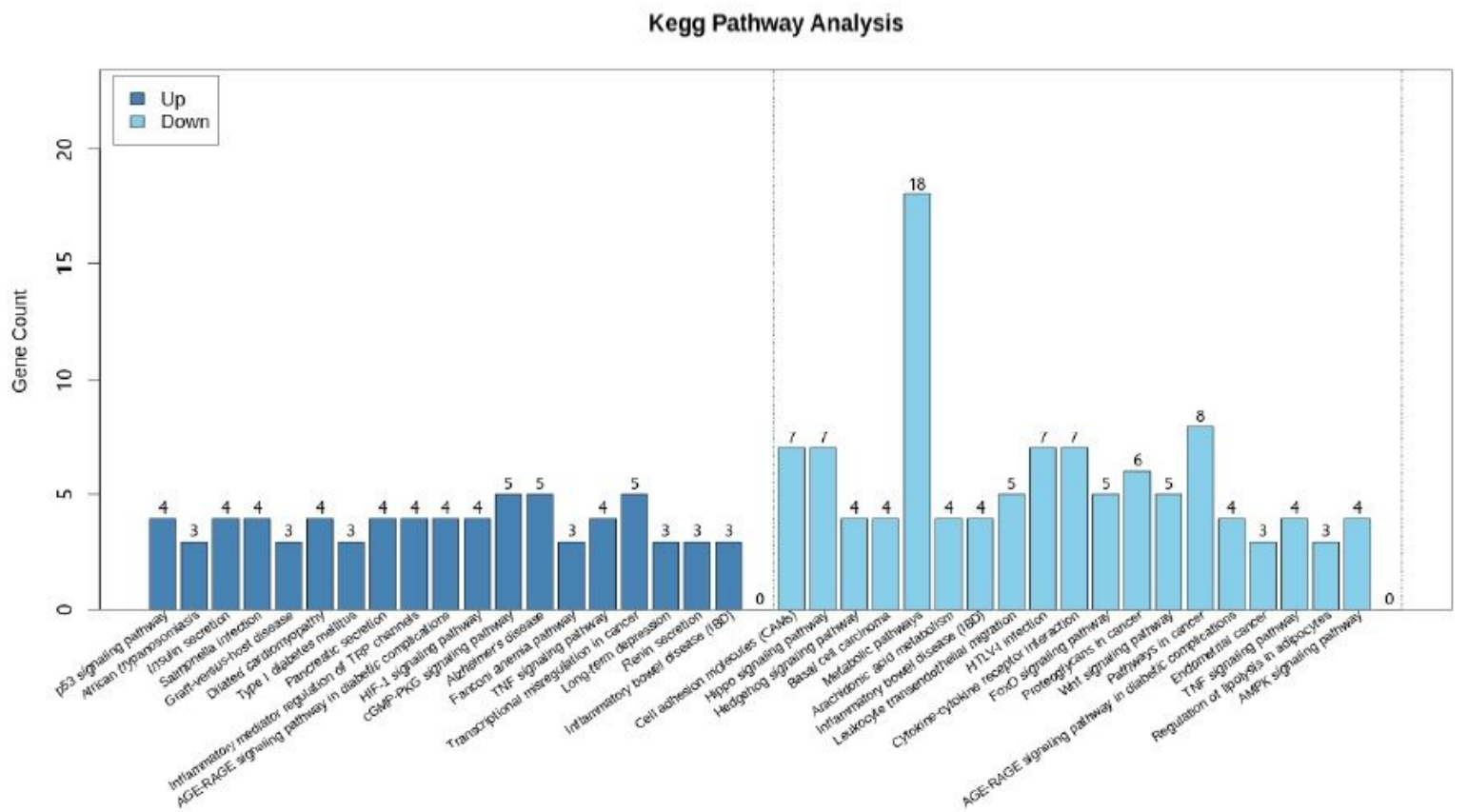

\section{Figure 4}

KEGG analysis of differentially expressed genes in the $0-10 \mu \mathrm{M}$ group and $\mathrm{C}-10 \mu \mathrm{M}$ group. $0-10 \mu \mathrm{M}$ group is the oocytes treated with $10 \mu \mathrm{M}$ of LPA and C-10 $\mu \mathrm{M}$ group is the cumulus cells treated with $10 \mu \mathrm{M}$ of LPA. 4A. KEGG analysis of upregulated and downregulated genes in the C-10 $\mu \mathrm{M}$ group; 4B. KEGG analysis of upregulated and downregulated genes in the 0-10 $\mu \mathrm{M}$ group; LPA, Lysophosphatidic acid; KEGG, Kyoto Encyclopedia of Genes and Genomes. 